\title{
Nanophotonics
}

SPIEDigitalLibrary.org/jnp

\section{Optical amplification of surface plasmon polaritons: review}

Kristjan Leosson 


\title{
Optical amplification of surface plasmon polaritons: review
}

\author{
Kristjan Leosson \\ Science Institute, University of Iceland, Dunhagi 3, IS107 Reykjavik, Iceland \\ kleos@hi.is
}

\begin{abstract}
Optical amplification of surface plasmon polaritons has been pursued extensively in recent years. However, few currently known optical gain materials can be expected to provide sufficient gain to fully compensate propagation losses of highly confined surface plasmon polaritons. Low-loss plasmonic waveguide geometries, on the other hand, provide a platform for realizing net plasmon gain by sacrificing some plasmonic properties. In search for potential applications for amplified surface plasmon polaritons, focus should be on making use of both optical gain and optical loss, such as in high-extinction-ratio modulators or parity-time symmetric devices. (C) 2012 Society of Photo-Optical Instrumentation Engineers (SPIE). [DOI: 10 .1117/1.JNP.6.061801]
\end{abstract}

Keywords: surface plasmon polaritons; integrated optical devices; optical gain; metal nanostructures; plasmon amplification.

Paper 12058VSS received Jun. 1, 2012; revised manuscript received Jul. 17, 2012; accepted for publication Jul. 25, 2012; published online Nov. 12, 2012.

\section{Introduction}

The study of collective electron excitations coupled with electromagnetic radiation on metaldielectric interfaces or in metal nanostructures (Fig. 1) has been a topic of intense research efforts, particularly during the past 10 to 15 years. The quanta of such excitations are the surface plasmon polaritons (SPPs), a term which nowadays is commonplace in the fields of nano-optics, bio-optics, photonics, photovoltaics, and quantum information processing. ${ }^{1-4}$ The rapidly increasing interest in plasmonics (the subfield of photonics involving metal surfaces and metallic nanostructures) has been, to a large degree, motivated by the desire to understand and control the behavior of light on the nanometer scale, and enabled by the great advances in micro and nanofabrication that have taken place during the past decades. The presence of surface plasmons in metallic nanostructures relates to a variety of unique optical effects, including extraordinary light transmission, giant field enhancement, negative refraction, and subwavelength waveguiding.

In contrast to dielectric photonic structures, dissipative losses are fundamental to SPPs, due to inelastic processes associated with the motion of conduction electrons in the metal. In some cases, this optical loss can be put to good use, e.g., for purposes of refractive index sensing with the surface-plasmon resonance (SPR) method $^{5}$ or in plasmonic nanoshell cancer treatment. ${ }^{6}$ However, in many cases, optical losses greatly limit the applicability of plasmonic technologies as a means to realizing, e.g., subwavelength photonic circuits or functional metamaterials at optical wavelengths. Consequently, the possibility of compensating or eliminating optical losses by introducing optical gain has also been actively investigated. The initial experiments of combining SPPs and gain materials date back to the late 1970s (Ref. 7) and 1980s (Ref. 8), but in recent years, there have been a number of important breakthroughs in this field, well documented by recent reviews on the topic. ${ }^{9,10}$

This paper will briefly cover some general aspects of loss and gain in plasmonic waveguides and nanoparticles, but will specifically discuss recent experimental and theoretical results on amplification in particular plasmonic waveguides that exhibit low optical loss, the so-called longrange surface plasmon polariton waveguides, ${ }^{11}$ as well as their newly proposed dielectric-loaded

0091-3286/2012/\$25.00 ㄷ 2012 SPIE 


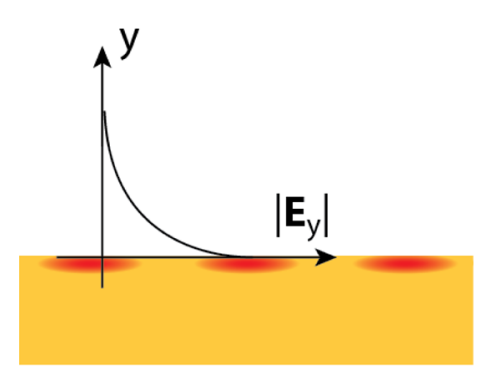

(a)

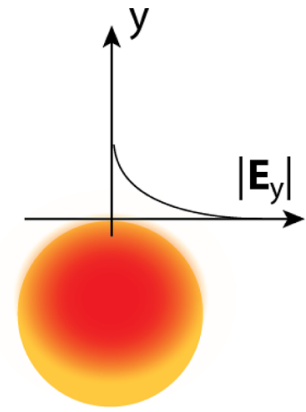

(b)

Fig. 1 (a) The surface plasmon polariton is associated with oscillations in the electron density at the metal-dielectric interface. (b) The localized surface plasmon of a metallic nanostructure is associated with oscillations in electron density of the whole nanostructure (fundamental dipolar mode of a spherical nanoparticle shown here).

counterparts. ${ }^{12}$ Possible applications of combining optical amplification and surface plasmon polariton waveguides are discussed.

\section{Loss and Gain in Plasmonic Waveguides and Nanoparticle SPASERS}

Many different metal-dielectric interface configurations have been considered as plasmonic waveguides, including planar interfaces, ridges, wedges, channels, wires, metal-dielectric-metal sandwich structures, and nanoparticle chains, as well as hybrid structures involving coupled dielectric and metallic waveguides, all exhibiting a different balance of field confinement versus optical loss. ${ }^{13-16}$ The material gain required to substantially compensate or overcome propagation losses in any waveguide system that provides a field confinement better than the conventional diffraction limit is quite significant, even by comparison with the best available gain materials. Gain figures ranging from 1000 to $80,000 \mathrm{~cm}^{-1}$ have been mentioned in the literature as requirements for lossless propagation. ${ }^{17-23}$

To put these numbers into perspective, it is instructive to look at the performance of currently available photonic devices that provide optical gain in the visible and near infrared range. For semiconductor lasers, a net modal gain of approximately $250 \mathrm{~cm}^{-1}$ is typical of state-of-the-art devices, operating around $1.5-\mu \mathrm{m}$ wavelength and similar values have been realized only very recently in rare-earth doped devices at $980 \mathrm{~nm} .{ }^{24}$ For organic gain media, net modal gains up to $100 \mathrm{~cm}^{-1}$ in slab waveguide geometries are reported in the visible range. ${ }^{25}$ Substantially higher material gain is often quoted for, e.g., quantum wells (QWs) or quantum dots (QDs). As an example, values up to $\approx 10^{5} \mathrm{~cm}^{-1}$ have been reported for saturation material gain in $1.3-\mu \mathrm{m}$ emitting InGaAs QDs, measured at $77 \mathrm{~K}$ (Ref. 26). However, due to inhomogeneous broadening and the low optical confinement factor (spectral and spatial overlap between the optical mode and the active gain material), this translates to a modal gain of only $40 \mathrm{~cm}^{-1}$, even in state-of-theart QD amplifiers. ${ }^{27}$ Using gain values from literature, Russev et al. ${ }^{28}$ found no materials that could provide sufficient effective gain to amplify SPPs on a single gold or aluminum surface, while simultaneously maintaining the surface-confinement of the SPP. On a silver-dielectric surface, however, they found that SPP net amplification should be possible using semiconductor QWs in the infrared region. Organic dyes providing $125 \mathrm{~cm}^{-1}$ effective gain at approximately $600-\mathrm{nm}$ wavelength came close to the required gain values. Garcia et al. ${ }^{29}$ obtained a $33 \%$ loss reduction in a dielectric-loaded SPP waveguide [Fig. 2(a)], corresponding to $143 \mathrm{~cm}^{-1}$ of optical gain, using QD-doped PMMA ridges. Based on a survey of the current literature, they claimed that further loss reduction in similar plasmonic waveguides was unrealistic with currently available gain materials, due to photobleaching and thermal damage at strong pump intensities.

From these considerations, it is clear that achieving lossless propagation or amplification of SPPs with field confinement below the diffraction limit represents a challenging task from the point of view of experiments and materials science, also suggesting that gain materials will have to be operated close to saturation levels and/or at cryogenic temperatures. Important advances 


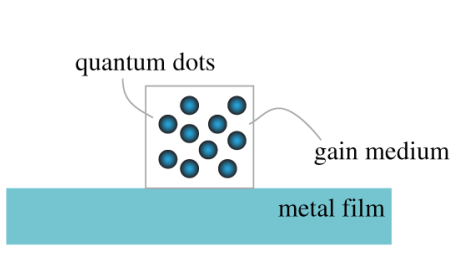

(a)

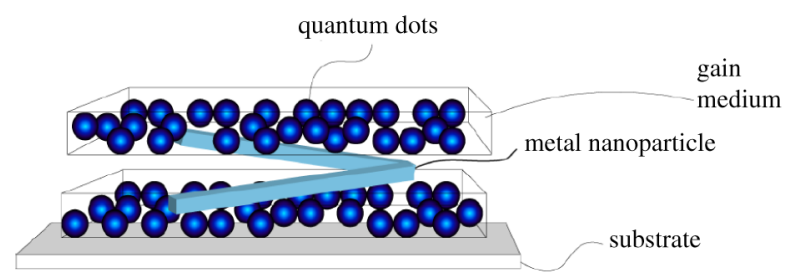

(b)

Fig. 2 (a) Example of a plasmonic waveguide with gain: cross-section of a dielectric-loaded surface plasmon polariton waveguide with a quantum-dot doped ridge. (b) A V-shaped nanoparticle SPASER, as originally proposed by Bergman and Stockman. The overall lateral size of the $\mathrm{V}$-shape is measured in $10 \mathrm{~s}$ of $\mathrm{nm}$ (reprinted by permission from M. Stockman).

have nevertheless been made in recent years with, e.g., metal-clad semiconductor laser structures and hybrid semiconductor-metal waveguide geometries, as has been reviewed in Ref. 10. Interestingly, a substantial increase (up to 1000-fold) in SPP modal gain was predicted to occur due to the slowing of the SPP wave for frequencies approaching the surface plasmon frequency in metal-dielectric-metal sandwich or coaxial geometries. ${ }^{30,31}$ This will serve to balance the increased losses at these wavelengths, even with effects of gain quenching taken into account ${ }^{31}$ and represents an interesting avenue for further studies of slow light amplification.

Individual metal particles have been considered as resonators for nanoscale plasmonic amplifiers (SPASERs), as proposed a decade ago by Bergman and Stockman. ${ }^{32}$ The SPASER was originally envisioned as consisting of a metal nanostructure [Fig. 2(b)] supporting localized plasmon resonances (structure size $<$ skin depth), although the term is nowadays often used also more broadly in the context of amplification of propagating SPP (in the latter case, the more appropriate term SPPASER has also been suggested). For the case of spherical gold or silver nanoparticles, the fundamental plasmon resonance has a lifetime of several fs (Ref. 33), corresponding well to that of strongly confined propagating SPPs. Simulations of metal particles coated with a dielectric shell have indicated that the active material must provide gain of the order of $10^{4} \mathrm{~cm}^{-1}$ to compensate losses in this mode, ${ }^{34}$ which is also of the same order as has been estimated for short-range surface plasma waves. Experimentally, spectrally narrow emission at $530 \mathrm{~nm}$ from gold nanoparticles coated with dye-doped glass (OG-488 in silica) has been observed ${ }^{35}$ but no independent determination of the material gain of the doped glass was provided. In general, the interaction between realistic gain materials and bright and dark plasmon modes of spherical metal nanoparticles ${ }^{36}$ and more complex nanostructures ${ }^{37,38}$ will certainly be an active topic of theoretical and experimental research in the coming years.

\section{Low-Loss Plasmonic Waveguide Geometries}

As pointed out in Sec. 2, values of modal gain observed in conventional gain materials (including semiconductors, conjugated polymers, dye-doped or QD-loaded matrices, and recently also in rare-earth doped crystals) are typically of the order of $10^{2} \mathrm{~cm}^{-1}$. Consequently, an experimentally feasible approach to realizing net gain in plasmonic waveguides is to reduce propagation losses below this value. Propagation losses are decreased by reducing the field-metal overlap, making the plasmon polariton mode more photon-like. This can be achieved by operating at a lower optical frequency ${ }^{39}$ and/or by changing the waveguide geometry. ${ }^{11,12}$ In either case, the plasmon-like properties of the SPP are weakened, meaning that the SPP wave vector, field confinement, and group velocity become closer to photonic values. Even though their propagation losses are usually still substantial, compared with purely dielectric waveguides, low-loss plasmonic waveguides have certain features that can be exploited for special applications, as discussed in Sec. 4, in addition to providing model systems for studying emitter-plasmon interactions.

The simplest low-loss plasmon waveguide geometry is represented by the thin metal slab with dielectric materials having similar or identical refractive index on either side [Fig. 3(a)]. Such a film supports coupled (symmetric and antisymmetric) TM-polarized SPP modes, corresponding to two single-interface SPPs in the thick-film limit. The low-loss (symmetric) mode is commonly referred 


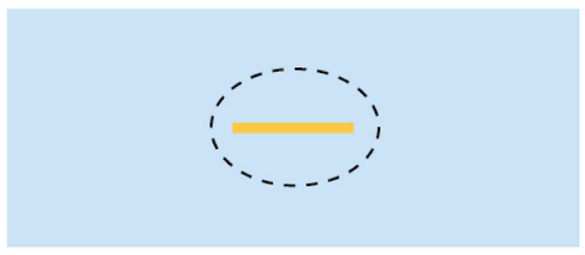

(a)

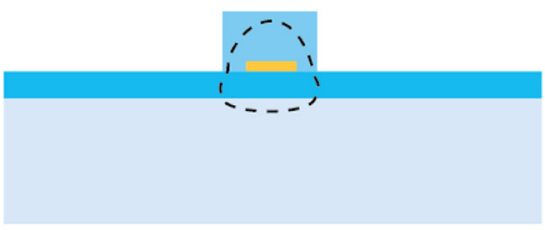

(b)

Fig. 3 Cross-sectional view of the (a) long-range surface plasmon polariton (LRSPP) waveguide geometry, and (b) the dielectric-loaded LRSPP waveguide geometry. The extent of the field of the long-range mode is indicated schematically with the dashed lines.

to as the long-range surface plasmon polariton (LRSPP) mode. ${ }^{11}$ For two-dimensional confinement, the metal slab can be patterned to form stripe waveguides that can be tailored in width and height, e.g., for close (>95\%) mode-matching to conventional single-mode optical fibers. Similarly, long-range modes supporting orthogonal polarizations can also be formed from the superposition of coupled plasmonic corner modes in rectangular metallic nanowire waveguides. ${ }^{40}$ Thin films, stripes, and nanowires also support short-range (antisymmetric) plasmon modes that provide strong field confinement (beyond what is possible to achieve on a single metal-dielectric interface), with a correspondingly increased propagation loss. This mode is important from the point of view of superfocusing, slow light propagation, surface-sensing, and strong light-matter interaction, including quenching of dipole lifetime and reduction of available LRSPP gain. ${ }^{41-44}$

Loss reduction of propagating LRSPPs at a wavelength of $1.53 \mu \mathrm{m}$ was first reported in 2008 (Ref. 45), using wafer-bonded Er-doped phosphate glass as gain material. Net gain in LRSPP waveguides was first demonstrated in 2010, using optical pumping (ns-pulses) of a conjugated fluorescent polymer providing gain at $600 \mathrm{~nm}$ (Ref. 46) or a liquid dye at $882 \mathrm{~nm}$ (Ref. 47). In these studies, plasmon propagation loss was reduced sufficiently by using a low LRSPP waveguide thickness $(5 \mathrm{~nm})$ or width $(1 \mu \mathrm{m})$, respectively. In both cases, the real part of the refractive index of the gain material was matched to that of the transparent substrate, as the gain material was introduced only on one side of the metal film. In 2011, net LRSPP gain was reported in an optically-pumped semiconductor multiple-QW structure at $1.46 \mu \mathrm{m}$ (Ref. 48), where the authors also estimated that the current density required to reach the gain threshold with electrical pumping would exceed only slightly the typical maximum values used for conventional laser diodes. All of the measurements were carried out at room temperature. As an alternative to conventional electrical pumping of a light-emitting p-n diode structure, Fedyanin et al. have suggested direct minority carrier injection in a Schottky (metal-semiconductor) diode as a means of amplifying long-wavelength surface plasmons. ${ }^{49}$ Such devices, however, remain to be investigated experimentally.

A low-loss variant of the dielectric-loaded plasmonic waveguide geometry was recently proposed and demonstrated experimentally. ${ }^{12}$ and ${ }^{50}$ In this case, a thin metal stripe waveguide is placed between a dielectric ridge and a high-index dielectric slab (buffer layer) supported by a lower-index dielectric substrate [Fig. 3(b)]. Calculated propagation lengths are of the order of millimeters at $1.5 \mu \mathrm{m}$ wavelength for mode confinement corresponding to 2 to 3 times the diffraction limit [taken as $\left.\lambda /\left(2 \mathrm{n}_{\text {ridge }}\right)\right]$. Introduction of optical gain into such a structure has also been considered using finite-difference calculations, ${ }^{51}$ revealing that material gain as low as 3 to $10 \mathrm{~cm}^{-1}$ is sufficient for lossless propagation of similarly confined plasmons at $1.5 \mu \mathrm{m}$, depending on whether gain is introduced in the ridge or the buffer layer. Even though dimensional tolerances will become more critical, it will be interesting to see this work extended toward the visible wavelength range.

\section{Potential Applications of Plasmonic Waveguides with Gain}

Plasmonic waveguides have many interesting properties that set them apart from dielectric waveguides. This certainly provides a rich playground for studies of light-matter interaction at the nanoscale. In the strongly plasmonic SPP regime, however, optical losses are prohibitively large for many practical applications, and loss compensation with optical gain will in many cases 
be energetically and economically unfeasible when it comes to designing functional devices. In the photon-like SPP regime, losses are lower but SPP waveguide properties become similar to dielectric waveguides. Currently, the most successful applications of plasmonics are those where the optical loss is used as an advantage, such as in SPR sensing and photo-thermal conversion, ${ }^{5,6}$ and those involving single-component nano-devices such as in plasmon-assisted magnetic recording and beam shaping. ${ }^{52}$

In searching for potential uses of SPP waveguides, it is therefore advisable to focus on applications that turn their limitations into strengths. A limiting factor in use of SPP-based devices in optical communications networks, for example, is the fact that SPP waveguide components are generally single-polarization devices. Polarization in the fiber network is generally unknown and varying irregularly with time, making polarization independence of optical components critical. However, this can be turned to an advantage in cases where it is important to eliminate polarization scattering, such as in quantum optical circuits. SPPs are suitable quantum information carriers, in spite of their optical losses ${ }^{53}$ For LRSPP waveguides, single-polarization operation and the high precision that can be achieved in patterning the waveguide core layer can contribute to high performance of LRSPP-based quantum optical circuits.

Benisty et al. ${ }^{54}$ have suggested the use of plasmonic waveguides for PT-symmetric optical devices with $\varepsilon_{(x)}=\varepsilon_{(-x)}$ * i.e., the optical analog of quantum-mechanical parity-time symmetric potentials. A schematic cross-section of a proposed device structure containing an LRSPP waveguide (for loss) and a dielectric waveguide (with gain) is shown in Fig. 4(a). Plasmonic waveguides with tunable optical loss provide a highly suitable platform for realizing such devices. Plasmon amplification in metal-insulator-metal structures [Fig. 4(b)] may serve to realize fast and ultracompact optical switches with a high extinction ratio $(>50 \mathrm{~dB}$, partly arising from large propagation losses in the off-state) that may be incorporated into a semiconductor waveguide platform. ${ }^{55}$

Properties of plasmonic waveguides are promising for suppression of lasing action in superluminescent diodes [Fig. 4(c)], thus improving the power-linewidth product compared with dielectric waveguide devices, ${ }^{56}$ mainly due to an increased spontaneous emission factor, compared with corresponding dielectric waveguide devices. Conversely, De Leon and Berini ${ }^{57}$ identified SPP-gain configurations with decreased spontaneous emission into the guided mode as potential low-noise amplifiers. These and other utilizations of radiative decay engineering in plasmonic systems will likely lead to interesting future applications.

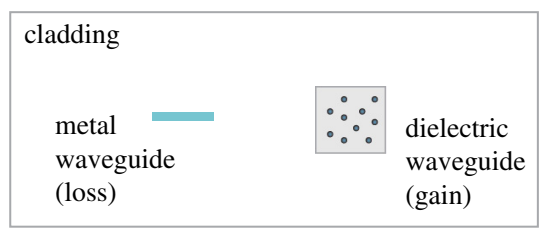

(a)

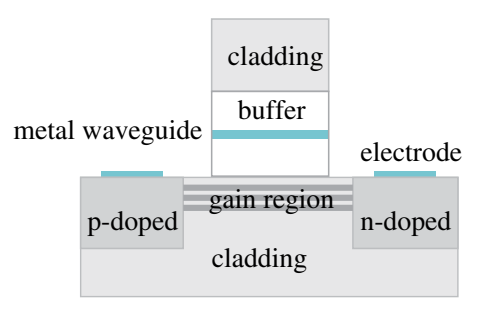

(c)

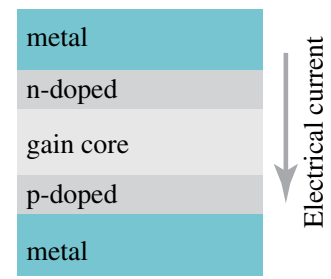

(b)

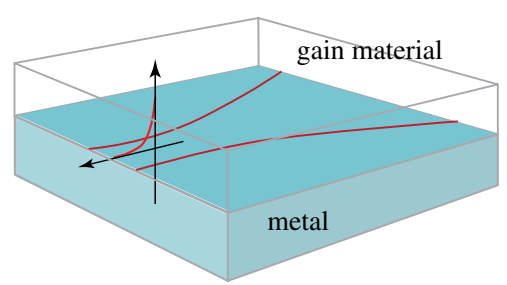

(d)

Fig. 4 Fig. 4. Potential applications of SPP loss/gain structures. (a) PT-symmetric optical device based on a directional coupler structure containing a lossy plasmonic waveguide and a dielectric waveguide with corresponding optical gain (adapted from Ref. 54). (b) Electrically driven metalsemiconductor-metal plasmonic modulator providing high extinction ratio and fast switching in an ultracompact device (adapted from Ref. 55). (c) Superluminescent diode structure with a plasmonic waveguide for lasing suppression (adapted from Ref. [56]). (d) Top view of a proposed low-noise optical amplifier with a focused Gaussian SPP beam (adapted from Ref. 56). 


\section{Conclusion}

Numerous recent theoretical and experimental studies have focused on incorporating optical gain into a dielectric material adjacent to a metal surface, to reduce or overcome absorption losses, realize plasmonic amplifiers, SPASERs, lossless metamaterials, and to further our understanding of spontaneous and stimulated emission from excited dipoles in the vicinity of a metal surface or metallic nanoparticles, for radiative decay engineering, fluorescence enhancement, biosensing, and nonlinear optical interactions. This field represents an exciting new area of research in plasmonics and light-matter interaction in general, even though plasmon losses are generally too high to allow for full loss compensation with currently available gain materials and reasonable pump power. Low-loss plasmonic waveguide geometries, however, have been shown to provide a suitable platform for the study of net plasmon amplification, using a range of currently available gain materials, with potential future applications in photonics.

\section{References}

1. S. Lal, S. Link, and N. J. Halas, "Nano-optics from sensing to waveguiding," Nat. Photon. 1(11), 641-648 (2007), http://dx.doi.org/10.1038/nphoton.2007.223.

2. D. K. Gramotnev and S. I. Bozhevolnyi, "Plasmonics beyond the diffraction limit," Nat. Photon. 4(2), 83-91 (2010), http://dx.doi.org/10.1038/nphoton.2009.282.

3. J. A. Schuller et al., "Plasmonics for extreme light concentration and manipulation," Nat. Mater. 9(3), 193-204 (2010), http://dx.doi.org/10.1038/nmat2630.

4. Z. Jacob and V. M. Shalaev, "Plasmonics goes quantum," Science 334(6055), 463-464 (2011), http://dx.doi.org/10.1126/science.1211736.

5. J. I. Homola and J. Dostálek, Surface plasmon resonance based sensors, Springer Series on Chemical Sensors and Biosensors, xii, p. 251, Springer, Berlin, New York (2006).

6. R. Bardhan et al., "Theranostic nanoshells: from probe design to imaging and treatment of cancer," Accounts Chem. Res. 44(10), 936-946 (2011), http://dx.doi.org/10.1021/ ar200023x.

7. G. A. Plotz, H. J. Simon, and J. M. Tucciarone, "Enhanced total reflection with surfaceplasmons," J. Opt. Soc. Am. 69(3), 419-421 (1979), http://dx.doi.org/10.1364/JOSA.69 .000419 .

8. A. N. Sudarkin and P. A. Demkovich, "Excitation of surface electromagnetic waves on the boundary of a metal with an amplifying medium," Sov. Phys. Uspekhi 34(7), 764-766 (1989).

9. P. Berini and I. De Leon, "Surface plasmon-polariton amplifiers and lasers," Nat. Photon. 6(1), 16-24 (2012), http://dx.doi.org/10.1038/nphoton.2011.285.

10. R. F. Oulton, "Surface plasmon lasers: sources of nanoscopic light," Mater. Today 15(1-2), 26-34 (2012), http://dx.doi.org/10.1016/S1369-7021(12)70018-4.

11. P. Berini, "Long-range surface plasmon polaritons," Adv. Opt. Photon. 1(3), 484-588 (2009), http://dx.doi.org/10.1364/AOP.1.000484.

12. T. Holmgaard, J. Gosciniak, and S. I. Bozhevolnyi, "Long-range dielectric-loaded surface plasmon-polariton waveguides," Opt. Express 18(22), 23009-23015 (2010), http://dx.doi .org/10.1364/OE.18.023009.

13. R. F. Oulton et al., "Confinement and propagation characteristics of subwavelength plasmonic modes," New J. Phys. 10(10), 105018 (2008), http://dx.doi.org/10.1088/1367-2630/ 10/10/105018.

14. P. Berini, "Figures of merit for surface plasmon waveguides," Opt. Express 14(26), 13030-13042 (2006), http://dx.doi.org/10.1364/OE.14.013030.

15. J. Grandidier et al., "Dielectric-loaded surface plasmon polariton waveguides: Figures of merit and mode characterization by image and Fourier plane leakage microscopy," Phys. Rev. B 78(24), 245419 (2008), http://dx.doi.org/10.1103/PhysRevB.78.245419.

16. H. Benisty and M. Besbes, "Plasmonic inverse rib waveguiding for tight confinement and smooth interface definition," J. Appl. Phys. 108(6), 063108 (2010), http://dx.doi.org/10 $.1063 / 1.3478746$. 
17. I. Avrutsky, "Surface plasmons at nanoscale relief gratings between a metal and a dielectric medium with optical gain," Phys. Rev. B 70(15), 155416-1-155416-6 (2004), http://dx.doi .org/10.1103/PhysRevB.70.155416.

18. M. P. Nezhad, K. Tetz, and Y. Fainman, "Gain assisted propagation of surface plasmon polaritons on planar metallic waveguides," Opt. Express 12(17), 4072-4079 (2004), http://dx.doi.org/10.1364/OPEX.12.004072.

19. X. J. Zhang et al., "Gain-assisted propagation of surface plasmon polaritons via electrically pumped quantum wells," Opt. Lett. 35(18), 3075-3077 (2010), http://dx.doi.org/10.1364/ OL.35.003075.

20. M. A. Noginov et al., "Compensation of loss in propagating surface plasmon polariton by gain in adjacent dielectric medium," Opt. Express 16(2), 1385-1392 (2008), http://dx.doi .org/10.1364/OE.16.001385.

21. S. A. Maier, "Gain-assisted propagation of electromagnetic energy in subwavelength surface plasmon polariton gap waveguides," Opt. Commun. 258(2), 295-299 (2006), http://dx .doi.org/10.1016/j.optcom.2005.07.064.

22. X. Chen et al., "Multi-level multi-thermal-electron FDTD simulation of plasmonic interaction with semiconducting gain media: applications to plasmonic amplifiers and nano-lasers," Opt. Express 18(16), 17220-17238 (2010), http://dx.doi.org/10.1364/OE.18.017220.

23. R. J. Rao and T. T. Tang, "Study on active surface plasmon waveguides and design of a nanoscale lossless surface plasmon waveguide," J. Opt. Soc. Am. B-Opt. Phys. 28(5), 1258-1265 (2011), http://dx.doi.org/10.1364/JOSAB.28.001258.

24. D. Geskus et al., "Giant optical gain in a rare-earth-ion-doped microstructure," Adv. Mater. 24(10), Op19-Op22 (2012), http://dx.doi.org/10.1002/adma.201101781.

25. S. Chenais and S. Forget, "Recent advances in solid-state organic lasers," Polym. Int. 61(3), 390-406 (2012), http://dx.doi.org/10.1002/pi.3173.

26. D. Bimberg et al., "Quantum dot lasers: breakthrough in optoelectronics," Thin Solid Films 367(1-2), 235-249 (2000), http://dx.doi.org/10.1016/S0040-6090(00)00697-0.

27. A. Salhi et al., "Enhanced performances of quantum dot lasers operating at $1.3 \mathrm{mu} \mathrm{m}$," IEEE J. Selected Top. Quant. Elect. 14(4), 1188-1196 (2008), http://dx.doi.org/10.1109/JSTQE .2008 .916182 .

28. S. C. Russev, G. G. Tsutsumanova, and A. N. Tzonev, "Conditions for loss compensation of surface plasmon polaritons propagation on a metal/gain medium boundary," Plasmonics 7(1), 151-157 (2012), http://dx.doi.org/10.1007/s11468-011-9288-2.

29. C. Garcia et al., "Partial loss compensation in dielectric-loaded plasmonic waveguides at near infra-red wavelengths," Opt. Express 20(7), 7771-7776 (2012), http://dx.doi.org/10 $.1364 /$ OE.20.007771.

30. D. B. Li and C. Z. Ning, "Giant modal gain, amplified surface plasmon-polariton propagation, and slowing down of energy velocity in a metal-semiconductor-metal structure," Phys. Rev. B 80(15), 153304 (2009), http://dx.doi.org/10.1103/PhysRevB.80.153304.

31. A. A. E. Saleh and J. A. Dionne, "Waveguides with a silver lining: Low threshold gain and giant modal gain in active cylindrical and coaxial plasmonic devices," Phys. Rev. B 85(4), 045407 (2012), http://dx.doi.org/10.1103/PhysRevB.85.045407.

32. D. J. Bergman and M. I. Stockman, "Surface plasmon amplification by stimulated emission of radiation: Quantum generation of coherent surface plasmons in nanosystems," Phys. Rev. Lett. 90(2), 027402 (2003), http://dx.doi.org/10.1103/PhysRevLett.90.027402.

33. S. A. Maier, Plasmonics : fundamentals and applications, p. 223, Springer. xxiv, New York (2007).

34. J. A. Gordon and R. W. Ziolkowski, "The design and simulated performance of a coated nano-particle laser," Opt. Express 15(5), 2622-2653 (2007), http://dx.doi.org/10.1364/OE .15 .002622 .

35. M. A. Noginov et al., "Demonstration of a spaser-based nanolaser," Nature 460(7259), 1110-U68 (2009), http://dx.doi.org/10.1038/nature08318.

36. G. C. D. Francs et al., "Mie plasmons:modes volumes, quality factors, and coupling strengths (Purcell factor) to a dipolar emitter," Int. J. Opt. 2012, 175162 (2012), http:// dx.doi.org/10.1155/2012/175162. 
37. M. I. Stockman, "Spasers explained," Nat. Photon. 2(6), 327-329 (2008), http://dx.doi.org/ 10.1038/nphoton.2008.85.

38. W. S. Chang et al., "Low absorption losses of strongly coupled surface plasmons in nanoparticle assemblies," Proc. Natl. Acad. Sci. U.S.A. 108(50), 19879-19884 (2011), http://dx .doi.org/10.1073/pnas.1113563108.

39. C. Sirtori et al., "Long-wavelength (lambda approximate to 8-11.5 mu m) semiconductor lasers with waveguides based on surface plasmons," Opt. Lett. 23(17), 1366-1368 (1998), http://dx.doi.org/10.1364/OL.23.001366.

40. J. Jung, T. Sondergaard, and S. I. Bozhevolnyi, "Theoretical analysis of square surface plasmon-polariton waveguides for long-range polarization-independent waveguiding," Phys. Rev. B 76(3), 035434 (2007), http://dx.doi.org/10.1103/PhysRevB.76.035434.

41. R. Y. Wan, F. Liu, and Y. D. Huang, "Ultrathin layer sensing based on hybrid coupler with short-range surface plasmon polariton and dielectric waveguide," Opt. Lett. 35(2), 244-246 (2010), http://dx.doi.org/10.1364/OL.35.000244.

42. T. Sondergaard and S. Bozhevolnyi, "Slow-plasmon resonant nanostructures: Scattering and field enhancements," Phys. Rev. B 75(7), 073402 (2007), http://dx.doi.org/10.1103/ PhysRevB.75.073402.

43. I. De Leon and P. Berini, "Theory of surface plasmon-polariton amplification in planar structures incorporating dipolar gain media," Phys. Rev. B 78(16), 161401 (2008), http://dx.doi.org/10.1103/PhysRevB.78.161401.

44. G. S. Winter, W. L Wedge, and W. L. Barnes, "Can lasing at visible wavelengths be achieved using the low-loss long-range surface plasmon-polariton mode?," New J. Phys. 8(80), 1-15 (2006), http://dx.doi.org/10.1088/1367-2630/8/8/125.

45. M. Ambati et al., "Observation of stimulated emission of surface plasmon polaritons," Nano Lett. 8(11), 3998-4001 (2008), http://dx.doi.org/10.1021/nl802603r.

46. M. C. Gather et al., "Net optical gain in a plasmonic waveguide embedded in a fluorescent polymer," Nat. Photon. 4(7), 457-461 (2010), http://dx.doi.org/10.1038/ nphoton.2010.121.

47. I. De Leon and P. Berini, "Amplification of long-range surface plasmons by a dipolar gain medium," Nat. Photon. 4(6), 382-387 (2010), http://dx.doi.org/10.1038/nphoton.2010.37.

48. R. A. Flynn et al., "A room-temperature semiconductor spaser operating near 1.5 mum," Opt. Express 19(9), 8954-61 (2011), http://dx.doi.org/10.1364/OE.19.008954.

49. D. Y. Fedyanin et al., "Surface plasmon polariton amplification upon electrical injection in highly integrated plasmonic circuits," Nano Lett. 12(5), 2459-2463 (2012), http://dx.doi .org/10.1021/n1300540x.

50. V. S. Volkov et al., "Long-range dielectric-loaded surface plasmon polariton waveguides operating at telecommunication wavelengths," Opt. Lett. 36(21), 4278-4280 (2011), http://dx.doi.org/10.1364/OL.36.004278.

51. S. M. Garcia-Blanco, M. Pollnau, and S. I. Bozhevolnyi, "Loss compensation in longrange dielectric-loaded surface plasmon-polariton waveguides," Opt. Express 19(25), 25298-25310 (2011), http://dx.doi.org/10.1364/OE.19.025298.

52. L. Novotny and N. van Hulst, “Antennas for light," Nat. Photon. 5(2), 83-90 (2011), http:// dx.doi.org/10.1038/nphoton.2010.237.

53. G. Di Martino et al., "Quantum statistics of surface plasmon polaritons in metallic stripe waveguides," Nano Lett. 12(5), 2504-2508 (2012), http://dx.doi.org/10.1021/ nl300671w.

54. H. Benisty et al., "Implementation of PT symmetric devices using plasmonics: principle and applications," Opt. Express 19(19), 18004-18019 (2011), http://dx.doi.org/10.1364/OE.19 .018004 .

55. V. E. Babicheva et al., "Plasmonic modulator based on gain-assisted metal-semiconductormetal waveguide," arXiv:1203.3374v1, (2012), http://dx.doi.org/10.1016/j.photonics.2012 .05 .008 .

56. M. Ranjbaran and X. Li, "Optimized dipole-surface plasmon waveguide coupling for enhancement of SLD performance," IEEE Photon. J. 2(5), 848-857 (2010), http://dx .doi.org/10.1109/JPHOT.2010.2072954. 
57. I. De Leon and P. Berini, "Theory of noise in high-gain surface plasmon-polariton amplifiers incorporating dipolar gain media," Opt. Express 19(21), 20506-20517 (2011), http://dx.doi.org/10.1364/OE.19.020506.

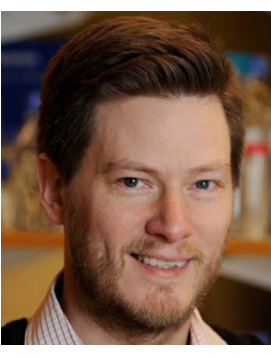

Kristjan Leosson received his $\mathrm{PhD}$ in electrical engineering from the Technical University of Denmark in 2002, where his research work focused on the optical properties of localized excitons in semiconductor nanostructures. Subsequently, he cofounded and worked for two start-up companies in Denmark, developing fabrication processes for integrated plasmonic circuits, for telecommunications and biotechnology. Since 2005, he has been employed as a senior research scientist at the Physics Department of the Science Institute of the University of Iceland. He has coauthored more than 50 peer-reviewed papers and is coinventor of four patents. 